\title{
Positron Emission Tomography in Renal Cell Carcinoma
}

\author{
(D) Cigdem Soydal MD'1, (1) Yüksel Ürün MD² \\ 'Ankara University Faculty of Medicine, Department of Nuclear Medicine, Ankara, Turkey \\ ${ }^{2}$ Ankara University Faculty of Medicine, Department of Internal Diseases, Division of Medical Oncology, Ankara, Turkey
}

\begin{abstract}
Renal cell carcinoma is the most common solid kidney tumor. Conventional methods such as computed tomography and magnetic resonance imaging are usually chosen for diagnosis, staging, and evaluating recurrence and treatment response. However, the sensitivity of these methods is limited in each indication. For this reason, metabolic evaluation with positron emission tomography has an important value in most solid tumors. However, the role of 18-fluorine-fluorodeoxyglucose (18F-FDG), which is the most commonly used positron emission tomography (PET) radiopharmaceutical, is limited in the evaluation of primary kidney lesions due to urinary excretion. Therefore, new radiopharmaceuticals with no or limited urinary excretion have been developed. In this paper, the application of PET imaging with the widely used 18F-FDG and the newly developed fluorothymidine and gallium-68/18F prostate-specific membrane antigen in renal cell carcinoma are reviewed.
\end{abstract}

Keywords: Renal cell carcinoma, positron emission tomography, 18F-fluorodeoxyglucose, 18F-fluorothymidine, gallium-68 prostate-specific membrane antigen

\section{Introduction}

Renal cell carcinoma (RCC) is the most common solid kidney tumor. Contrast-enhanced computed tomography (CT) is the most frequently used imaging modality in the diagnosis, staging, and evaluation of recurrence and treatment response in patients with RCC. The overall success rate of CT for these indications is reported to be between $61 \%$ and $91 \%(1,2,3)$. However, as RCCs may appear isodense, hypodense, or hyperdense, it is difficult to distinguish benign and malignant renal masses by morphological methods (4). Magnetic resonance imaging (MRI) is recommended in cases where CT is contraindicated, such as patients who have contrast allergy or are pregnant. However, $\mathrm{MRI}$ is no more accurate than CT. This increases the importance of positron emission tomography (PET), which enables metabolic evaluation in addition to visualizing anatomic changes. In this review, we discuss currently available literature data regarding the use of PET applications with different radiopharmaceuticals in patients with RCC.

\section{Applications of Positron Emission Tomography in Renal Cell Carcinoma}

18-Fluorine-Fluorodeoxyglucose Positron Emission Tomography

PET is a metabolic imaging method that utilizes various positron-emitting radiopharmaceutical and can provide data on many different metabolic pathways. The most widely used radiopharmaceutical is a fluorodeoxyglucose molecule (FDG) labeled with 18-fluorine (18F). The modality is based on the principle of visualizing elevated glycolysis and glucose uptake in neoplastic tissues. Despite high success rates in many solid organ malignancies, the use of 18F-FDG for urinary system malignancies is limited due to excretion via the urinary tract. The first cases related to the use of 18F-FDG PET in RCC were described by Wahl et al. (5) in the early 1990s. The use of $18 \mathrm{~F}-\mathrm{FDG}$ PET in the detection of primary RCC is especially controversial $(6,7,8,9,10)$. High and variable levels of background renal activity make it difficult to detect the primary focus. Forced diuresis with hydration may increase the sensitivity 
of 18F-FDG PET (11). However, many studies have shown that forced diuresis does not increase the sensitivity of 18F-FDG PET, and that background renal activity is actually increased in up to $60 \%$ of patients after diuretic injection due to physiological retention in the renal tubular epithelium $(11,12,13,14,15,16)$. In addition to background physiological activity in the kidney, sensitivity is also affected by the size of the primary tumor and the rate of $18 \mathrm{~F}-\mathrm{FDG}$ uptake. It has been shown that tumors exhibiting uptake on 18F-FDG PET are larger in size and contain more glucose transporter-1 (GLUT-1) receptor compared to tumors without uptake $(7,17,18)$. As a result, even when performed with forced diuresis, 18F-FDG PET is not an ideal imaging modality for diagnosing RCC. It is not possible to reach a conclusion about the place of 18F-FDG PET/CT in RCC diagnosis based on the limited information available in the literature (Table 1).

The introduction of hybrid PET/CT systems in routine practice has enabled more successful determination of tumor location. Sensitivity increases significantly in the detection of extrarenal lesions, although specificity does not change (19). Another advantage of the hybrid PET/CT system when monitoring for recurrence is the ability to differentially diagnose postoperative scar tissue, surgical clips, and displacement of the surrounding organs, which are difficult to distinguish in CT (20). Finally, 18F-FDG PET/CT allows whole-body assessment with a single imaging session, without the risk of contrast allergy or nephrotoxicity (21).

The quantitative evaluation of uptake using standard uptake value (SUV) has prognostic significance. Patients with higher SUV values at baseline are shown to have poorer prognosis and shorter survival. In addition, the presence of metastases may affect the mean SUV of the primary lesion. The mean SUV value of primary lesions of patients without distant organ metastasis was calculated as 2.6 , compared to 5.0 for patients with distant metastases (22). Besides SUV values, metabolic parameters such as metabolic tumor volume and total lesion glycolysis calculated with PET imaging also have prognostic significance $(23,24)$.

The sensitivity and specificity of 18F-FDG PET in the detection of extrarenal lesions have been reported as $79 \%$ and $90 \%$, respectively (25). Loss of sensitivity due to urinary excretion of FDG is not observed with extrarenal lesions. However, 18F-FDG PET cannot detect small lesions as well as it does large lesions. The sensitivity of FDG PET increases from $76 \%$ to $93 \%$ when lesion size increases from $1 \mathrm{~cm}$ to $2 \mathrm{~cm}$ (26). Furthermore, high-grade tumors are located more accurately than low-grade tumors (Table 2) $(19,21)$. 18F-FDG PET/CT has high sensitivity in detection of distant organ metastases and restaging RCC (Figures 1 and 2) (22). Another common indication for 18F-FDG PET/CT is evaluating response to tyrosine kinase inhibitor therapy. The Response Evaluation Criteria in Solid Tumors (RECIST) are frequently used when evaluating treatment response with anatomical methods such as CT and MRI. In this method, response is evaluated based on change in target lesion size. However, most antiangiogenic therapies used in recent years are cytostatic instead of cytotoxic, and usually cause tumor stabilization rather than tumor shrinkage. Another disadvantage of conventional methods is that they require a relatively long time for treatment response to be apparent radiologically. Many publications have reported that $18 \mathrm{~F}$ FDG PET/CT provides a more accurate assessment than the radiologic RECIST $(22,27,28,29)$. 18F-FDG PET/CT is particularly superior for assessing treatment response in bone metastasis because the RECIST describe soft tissue lesions (28).

\section{8-Fluorine-Fluorothymidine Positron Emission Tomography}

$18 \mathrm{~F}$-Fluorothymidine (FLT) is a thymidine analog that accumulates in the cell after phosphorylation by thymidine kinase but is not integrated into DNA structures (30). This radiopharmaceutical

Table 1. Diagnostic accuracy of 18-fluorine-fluorodeoxyglucose positron emission tomography/computed tomography for primary renal cell carcinoma lesions

\begin{tabular}{|l|l|l|l|l|l|l|}
\hline Authors & TP & FP & TN & FN & Sensitivity & Specificity \\
\hline Ramdave et al. (6) & 15 & 0 & 1 & 1 & 94 & 100 \\
\hline Miyakita et al. (18) & 6 & 0 & 13 & 0 & 32 & - \\
\hline Aide et al. (12) & 14 & 1 & 16 & 4 & 47 & 80 \\
\hline Kang et al. (8) & 9 & 0 & 6 & 2 & 60 & 100 \\
\hline TP: True positive, FP: False positive, TN: True negative, FN: False negative & \\
\hline
\end{tabular}

Table 2. Diagnostic accuracy of 18-fluorine-fluorodeoxyglucose positron emission tomography/computed tomography for extrarenal lesions

\begin{tabular}{|l|l|l|l|l|l|l|}
\hline Authors & TP & FP & TN & FN & Sensitivity & Specificity \\
\hline Ramdave et al. (6) & 2 & 0 & 0 & 15 & 100 & 100 \\
\hline Chang et al. (39) & 9 & 1 & 1 & 4 & 90 & 80 \\
\hline Aide et al. (12) & 10 & 3 & 0 & 40 & 100 & 93 \\
\hline Jadvar et al. (7) & 15 & 1 & 6 & 3 & 71 & 75 \\
\hline Majhail et al. (26) & 14 & 0 & 7 & 3 & 67 & 100 \\
\hline TP: True positive, FP: False positive, TN: True negative, FN: False negative
\end{tabular}




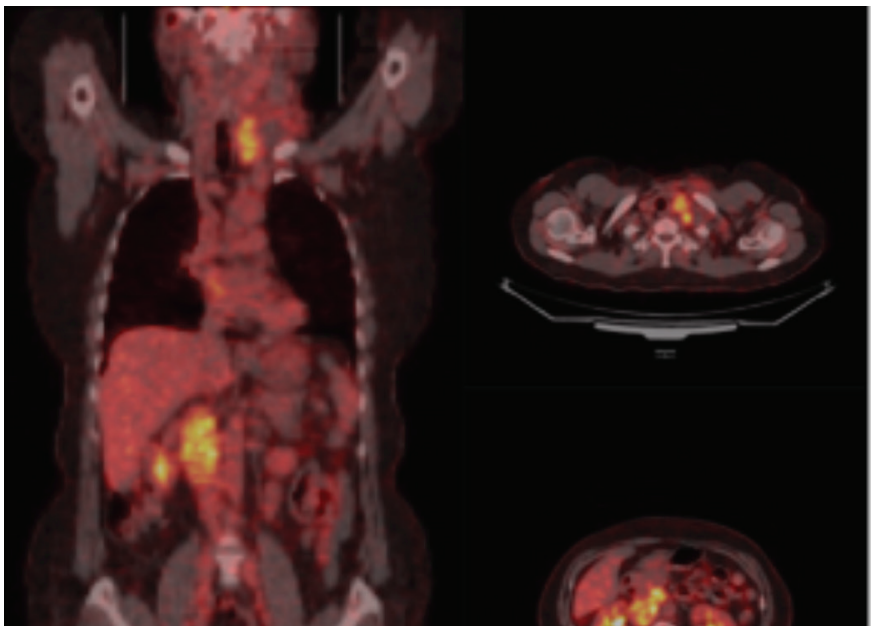

Figure 1. A 61-year-old female patient with history of renal cell carcinoma excision underwent 18-fluorine-fluorodeoxyglucose positron emission tomography/computed tomography for restaging of a soft tissue structure detected in the paraaortic area during follow-up. Pathologic uptake levels were noted in conglomerate lymph nodes filling the left supraclavicular and posterior cervical region $\left(\mathrm{SUV}_{\max }: 8.4\right)$ and in the abdominal lymph nodes in the paraaortic and retrocrural area $\left(S_{U V} V_{\max }: 10.3\right)$

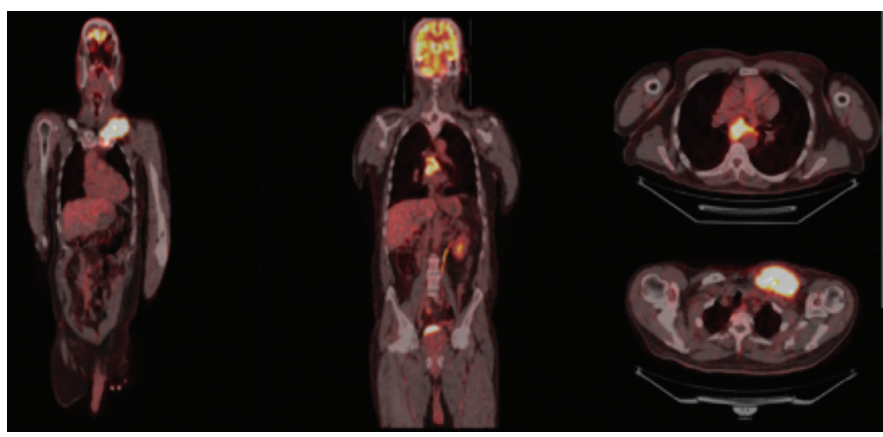

Figure 2. A 60-year-old male patient being under follow-up after right nephrectomy underwent 18-fluorine-fluorodeoxyglucose positron emission tomography/computed tomography due to detection of left supraclavicular and subcarinal lymph nodes on computed tomography. Intense pathologic 18-fluorinefluorodeoxyglucose uptake was seen in the left supraclavicular lymph nodes (SUV max $\left._{2}: 22.0\right)$ and subcarinal lymph nodes $\left(S_{\text {max }}\right.$ : 16.3)

has the potential to enable PET evaluation of tumor proliferation $(31,32)$. As a radiopharmaceutical that allows assessment of proliferation, its greatest advantage over 18F-FDG is the ability to more accurately distinguish inflammation and tumors. FLT uptake and higher initial FLT uptake are prognostic indicators in RCC (30). A correlation was shown between FLT uptake and Ki-67 index, a histopathologic proliferation marker (33). Although SUV values are lower than with 18F-FDG, metastatic lesions can be detected successfully because the physiological background activity is lower in regions such as the brain and mediastinum. However, it has a limited role in the assessment of liver metastases due to uptake associated with physiological activity in the liver. There are reports that FLT PET can be used successfully in the evaluation of treatment response in many tumors (34). Liuet al. (35) conducted FLT PET studies in 16 patients with RCC before sunitinib treatment, at the end of the first cycle of sunitinib treatment, and at the end of the washout period. Although they noted a marked decrease in proliferation during treatment, cell proliferation was markedly increased in the washout period. FLT PET allows evaluation of treatment response much earlier than FDG PET, even within the first week of therapy (36). Hybrid PET/MRI systems, which were recently introduced in clinical practice to assess treatment response of recurrence and solid organ metastases such as liver, are still used experimentally in many centers and have yielded encouraging results $(37,38)$.

\section{Gallium-68/18-Fluorine Prostate-Specific Membrane Antigen Positron Emission Tomography/Computed Tomography}

Prostate-specific membrane antigen (PSMA) is an antigenic molecule present on the surface of prostate cancer cells. It is also expressed in RCC and many solid tumors with tumor neovascularization $(39,40)$. PSMA is an exocrine expressed in the proximal tubule cells in normal kidney and to varying degrees in neovascular clear cell RCC (75\%), chromophobe RCC (31\%), oncocytoma (53\%), and transitional cell carcinoma (21\%) (40). PET imaging can be performed by binding PSMA to target molecules gallium (Ga)-68 or 18F. Ga-68 PSMA uptake in RCC was first reported as a case report (41). Histopathologic evidence indicates that while PSMA uptake does occur in areas of neovascularization, uptake in the proximal tubules occurs not in the adjacent vascular structures, but rather in the tubule cells (42). Moreover, it has been shown that PSMA expression is lost in RCCs arising from proximal tubule cells that express PSMA (40). In another case series, 18F PSMA uptake was assessed in 5 patients. In these five cases, 18F PSMA uptake was observed at varying levels with SUV values ranging from 1.6 to 19.3 in different metastatic lesions, and more metastatic foci were detected compared to conventional imaging methods (43). PSMA PET/CT seems to be particularly useful in patients with suspicious lesions detected by conventional imaging methods, such as when evaluating oligometastatic patients with subcentrimetric lesions or when identifying potentially resectable neighboring tumor foci in patients scheduled for cytoreductive nephrectomy (44). In addition to its high sensitivity, PSMA PET is also a functional imaging method that enables in vivo assessment of baseline neovascularization in metastatic lesions and may help predict treatment response prior to anti-vascular therapies such as tyrosine kinase inhibitors and bevacizumab. Another interesting finding is that sarcomatoid degeneration in RCCs is correlated with 18F-FDG uptake, not PSMA uptake, and histopathological samples show loss of PSMA expression and increased GLUT-1 receptor expression in sarcomatoid degeneration (45). The combined use of two PET studies may allow the non-invasive evaluation of sarcomatoid degeneration in different metastatic foci. Based on currently available data, PSMA PET seems likely to serve as a complementary method to enable detection of small metastatic foci undetectable by conventional methods and help evaluate response to treatments targeting neovascularization in future RCC patients. 
However, most of the data in the literature are from case series and small patient groups. Therefore, the results of prospective studies involving larger numbers of patients are needed.

\section{Conclusion}

PET studies using different radiopharmaceuticals can be performed with varying sensitivity in the diagnosis, staging, and evaluation of recurrence and treatment response in patients with RCC. The ability to detect primary tumoral lesions by $18 \mathrm{~F}-$ FDG PET is limited due to renal excretion. However, the success rate is high for metastatic foci. FLT PET, which demonstrates proliferation, and PSMA PET, which targets neovascularization, are also effective both in diagnosis and treatment response evaluation. In addition, high uptake at time of diagnosis is a prognostic indicator for all three pharmaceuticals.

\section{Ethics}

Peer-review: Externally peer-reviewed.

\section{Authorship Contributions}

Concept: C.S., Y.Ü., Design: Y.Ü., Data Collection or Processing: C.S., Analysis or Interpretation: C.S., Y.Ü., Literature Search: C.S., Writing: C.S., Y.Ü.

Conflict of Interest: No conflict of interest was declared by the authors.

Financial Disclosure: The authors declared that this study received no financial support.

\section{References}

1. Bechtold RE, Zagoria RJ. Imaging approach to staging of renal cell carcinoma. Urol Clin North Am 1997;24:507-522.

2. Levine E. Renal cell carcinoma: clinical aspects, imaging diagnosis, and staging. Semin Roentgenol 1995;30:128-148.

3. Zagoria RJ, Bechtold RE, Dyer RB. Staging of renal adenocarcinoma: role of various imaging procedures. AJR Am J Roentgenol 1995; 164:363-370.

4. Hilton S. Imaging of renal cell carcinoma. Semin Oncol 2000;27:150-159.

5. Wahl RL, Harney J, Hutchins G, Grossman HB. Imaging of renal cancer using positron emission tomography with 2-deoxy-2-(18F)-fluoro-Dglucose: pilot animal and human studies. JUrol 1991;146:1470-1474.

6. Ramdave S, Thomas GW, Berlangieri SU, et al. Clinical role of F- 18 fluorodeoxyglucose positron emission tomography for detection and management of renal cell carcinoma. J Urol 2001;166:825-830.

7. Jadvar H, Kherbache HM, Pinski JK, Conti PS. Diagnostic role of [F-18]-FDG positron emission tomography in restaging renal cell carcinoma. Clin Nephrol 2003;60:395-400.

8. Kang DE, White RL Jr, Zuger JH, et al. Clinical use of fluorodeoxyglucose F 18 positron emission tomography for detection of renal cell carcinoma. J Urol 2004;171:1806-1809.

9. Bachor R, Kotzerke J, Gottfried HW, et al. [Positron emission tomography in diagnosis of renal cell carcinoma]. Urologe A 1996;35:146-150.

10. Kumar R, Chauhan A, Lakhani P, et al. 2-Deoxy-2-[F-18] fluoroD-glucose-positron emission tomography in characterization of solid renal masses. Mol Imaging Biol 2005;7:431- 439.

11. Kamel EM, Jinchlinski P, Prior JO, et al. Forced diuresis impoves the diagnostic accuracy of 18F-FDG PET in abdominopelvic malignencies. J Nucl Med 2006;47:1803-1807.
12. Aide N, Cappele O, Bottet P, et al. Efficiency of [(18)F]FDG PET in characterising renal cancer and detecting distant metas-tases: a comparison with CT. Eur J Nucl Med Mol 2003;30: 1236-1245.

13. Ozülker T, Ozülker F, Ozbek E, Ozpaçaci T. A prospective diagnostic accuracy study of F-18 fluorodeoxyglucose-positron emission tomography/computed tomography in the evaluation of indeterminate renal masses. Nucl Med Commun 2011;32:265-272.

14. Southworth R, Parry CR, Parkes HG, et al. Tissue-specific differ- ences in 2-fluoro-2-deoxyglucose metabolism beyond FDG-6-P: a 19F NMR spectroscopy study in the rat. NMR Biomed 2003;16:494-502.

15. Thorens B. Glucose transporters in the regulation of intestinal, renal, and liver glucose fluxes. Am J Physiol 1996;270:541-553.

16. Montravers F, Grahek D, Kerrou K, et al. Evaluation of FDG uptake by renal malignancies (primary tumor or metastases) using a coincidence detection gamma camera. J Nucl Med 2000;41:78-84.

17. Park JW, Jo MK, Lee HM. Significance of 18F-fluorodeoxyglu- cose positron-emission tomography/computed tomography for the postoperative surveillance of advanced renal cell carcinoma. BJU Int 2009;103:615-619.

18. Miyakita $\mathrm{H}$, Tokunaga $\mathrm{M}$, Onda $\mathrm{H}$, et al. Significance of $18 \mathrm{~F}-\mathrm{FDC}$ flourodeoxyglucose positron emission tomography (FDG-PET) for detection of renal cell carcinoma and immunohistochemical glucose transporter 1 (GLUT-1) expression in the caner. Int J Urol 2002;9:15-18.

19. Kumar R, Shandal V, Shamim SA, et al. Role of FDG PET-CT in recurrent renal cell carcinoma. Nucl Med Commun 2010;31:844-850.

20. Tehrani OS, Shields AF. PET imaging of proliferation with pyrimidines. J Nucl Med 2013;54:903-912.

21. Sacco E, Pinto F, Totaro A, et al. Imaging of renal cell carcinoma: state of the art and recent advances. Urol Int 2011;86:125-139.

22. Liu Y. The palce of FDG PET/CT in renal cell carcinoma: Value and Limitations. Front Oncol 2016;6:201.

23. Hwang SH, Cho A, Yun M, et al. Prognostic Value of Pretreatment Metabolic Tumor Volume and Total Lesion Glycolysis Using 18F-FDG PET/CT in Patients With Metastatic Renal Cell Carcinoma Treated With Anti-Vascular Endothelial Growth Factor-Targeted Agents. Clin Nucl Med 2017;42:235-241.

24. Nakajima R, Matsuo Y, Kondo T, et al. Prognostic Value of Metabolic Tumor Volume and Total Lesion Glycolysis on Preoperative 18F-FDG PET/CT in Patients With Renal Cell Carcinoma. Clin Nucl Med 2017;42:177-182.

25. Wang HY, Ding HJ, Chen JH, et al. Meta-analysis of the diagnostic performance of 18F-FDG-PET and PET/CT in renal cell carcinoma, Cancer Imaging 2012;12:464-474.

26. Majhail NS, Urbain JL, Albani JM, et al. F-18 fluorodeoxyglucose positron emission tomography in the evaluation of distant metastases from renal cell carcinoma. J Clin Oncol 2003;21:3995-4000.

27. Kayani I, Avril N, Bomanji J, et al. Sequential FDG-PET/CT as a biomarker of response to Sunitinib in metastatic clear cell renal cancer. Clin Cancer Res 2011;17:6021-6028.

28. Lyrdal D, Boijsen $M$, Suurküla $M$, et al. Evaluation of sorafenib treatment in metastatic renal cell carcinoma with 2-fluoro-2deoxyglucose positron emission tomography and computed tomography. Nucl Med Commun 2009;30:519-524.

29. Kakizoe M, Yao M, Tateishi U, et al. The early response of renal cell carcinoma to tyrosine kinase inhibitors evaluated by FDG PET/CT was not influenced by metastatic organ. BMC Cancer 2014;14:390.

30. Park JW, Jo MK, Lee HM. Significance of 18F-fluorodeoxyglu- cose positron-emission tomography/computed tomography for the postoperative surveillance of advanced renal cell carcinoma. BJU Int 2009;103:615-619.

31. Shields AF, Briston DA, Chandupatla $S$, et al. A simplified analysis of [18 F]3'-deoxy-3'-fluorothymidine metabolism and retention. Eur J Nucl Med Mol Imaging 2005;32:1269-1275. 
32. Shields AF, Grierson JR, Dohmen BM, et al. Imaging proliferation in vivo with [F-18]FLT and positron emission tomography. Nat Med 1998;4:1334-1336.

33. Wong PK, Lee ST, Murone C, et al. In vivo imaging of cellular proliferation in renal cell carcinoma using $18 \mathrm{~F}$-fluorothymidine PET. Asia Ocean J Nucl Med Biol 2014;2:3-11.

34. Sanghera $B$, Wong WL, Sonoda LI, et al. FLT PET-CT in evaluation of treatment response. Indian J Nucl Med 2014;29:65-73.

35. Liu G, Jeraj R, Vanderhoek M et al. Pharmacodynamic study using FLT $\mathrm{PET} / \mathrm{CT}$ in patients with renal cell cancer and other solid malignancies treated with sunitinib malate. Clin Cancer Res 2011;17:7634-44.

36. Horn KP, Yap JT, Agarwal N, et al. FDG and FLT-PET for Early measurement of response to $37.5 \mathrm{mg}$ daily sunitinib therapy in metastatic renal cell carcinoma. Cancer Imaging 2015;15:15.

37. Antunes J, Viswanath S, Rusu M, et al. Radiomics Analysis on FLT-PET/ MRI for Characterization of Early Treatment Response in Renal Cell Carcinoma: A Proof-of-Concept Study. Transl Oncol 2016;9:155-162.

38. Valls $L$, Hoimes $C$, Sher $A$, et al. Early response monitoring of receptor tyrosine kinase inhibitor therapy in metastatic renal cell carcinoma using [F-18]fluorothymidine-positron emission tomographymagnetic resonance. Semin Roentgenol 2014;49:238-241.
39. Chang SS, O'Keefe DS, Bacich DJ, et al. Prostate-specific membrane antigen is produced in renal neoplasms. Clin Cancer Res 1999;5:219-227.

40. Baccalae A, Sercia L, Li J, et al. Expression of prostate specific membrane antigen in tumor-associated neovasculature of renal neoplasm. Urology 2007;70:385-390.

41. Demirci E, Ocak M, Kabasakal L, et al. (68) Ga-PSMA PET/CT imaging of metastatic clear cell renal carcinoma. Eur J Nucl Med Mol Imaging 2014;41:1461-1462.

42. Rhee $\mathrm{H}, \mathrm{Ng} \mathrm{KL}$, Tse BW, et al. Using prostate specific membrane antigen (PSMA) expression in clear cell renal cell carcinoma for imaging advanced disease. Pathology 2016;48:613-616.

43. Rowe SP, Gorin MA, Hammers HJ, et al. Imaging of metastatic clear cell renal cell carcinoma with PSMA-targeted ${ }^{18} \mathrm{~F}$-DCFPyL PET/CT. Ann Nucl Med 2015;29:877-882.

44. Rhee H, Blazak J, Tham CM, et al. Pilot study: use of gallium-68 PSMA PET for detection of metastatic lesions in patients with renal tumour. EJNMMI Res 2016;6:76.

45. Nadebaum DP, Hofman MS, Mitchell CA, et al. Oligometastatic Renal Cell Carcinoma With Sarcomatoid Differentiation Demonstrating Variable Imaging Phenotypes on 68Ga-PSMA and 18F-FDG PET/CT: A Case Report and Review of the Literature. Clin Genitourin Cancer 2017. 